\title{
IL TERREMOTO DELLO HOKKAIDO DEL 4 MARZO 1952
}

\section{Antonino Girlanda}

\section{Parte II}

Determinazione della dromocrona più probabile delle onde $\mathrm{P}_{\mathrm{n}}$ e suo confronto con $i$ tempi calcolati da altri ricercatori.

In una nota precedente (') sono state determinate le coordinate epicentrali e l'ora origrine del terremoto dello Hokkaido del 4 marzo 1952. Si è ottenuto:

$$
\begin{aligned}
& \text { i. }=+143^{\prime \prime} 30^{\prime}\left(00^{\prime \prime}, 3 \pm 03^{\prime} 35^{\prime \prime}, 4,\right. \\
& \text { 小 }+42^{\prime} 14^{\prime} 30^{\prime \prime}, 8 \pm 04^{\prime} 19^{\prime \prime}, 5 \text { (latitudine geocentrica), } \\
& t_{0}=01^{11} 22^{\mathrm{m}} 47^{\mathrm{s}}, 5 \pm 00^{\mathrm{s}}, 3 .
\end{aligned}
$$

Una prima serie di calcoli, condotti seguendo il metodo di CaloiPeronaci $(\because$, ha escluso la possililita di una profondità apprezzabile.

Servendosi degli elementi grià calcolati si determinerà la dromocrona più probal,ile delle $P$, valevole per il terremoto oggetto di questo studio, allo scopo di poter trarre elementi di giudizio relativamente ad alcune discordanze notate nei tempi di registrazione degli inizi in alcune slazioni.

Si ammelle che l'equazione della dromocrona delle $P$ si possa porre solto la forma approssimata

$$
t=\alpha_{0}+\alpha_{1} \Delta+\alpha_{2} \Delta^{2}+\alpha_{3} \Delta^{3}
$$

Nella quale $t$ i il tempo di tragritto, $\perp$ la distanza epicentrale e $a_{0}, a_{1}, a_{0}, u_{3}$, sono coefficienti, indipendenti da $\Delta$, da determinare in arlattamento ai dati sperimentali. Denotando con $T_{\mathrm{i}}$ il tempo di arrivo delle $P$ nella $i$-esima stazione di osservazione, la [1] fornisce il sistema di $n$ equazioni :

$$
T_{\mathrm{i}}-t_{0}=\alpha_{0}+\alpha_{1} \Delta_{\mathrm{i}}+\alpha_{2} \Delta_{\mathrm{i}}^{2}+\alpha_{3} \Delta_{\mathrm{i}}^{3}
$$

nelle quattro incognite $\omega_{0}, \alpha_{1}, u_{0}, u_{0}$, la determinazione delle quali si puó effeltuare col metodo dei minimi quadrati, qualora si disponga dei tempi $T_{2}$ in un sufficiente numero di stazioni. 
Per rendere meno laborioso il calcolo si può procedere nel modo seguente.

Dalle [2], scritte, per es., in ordine di distanze crescenti, si deducono per differenza le $n \cdot 1$ equazioni:

$$
\delta_{i+1, i}=\alpha_{1}\left(\Delta_{i+1}-\Delta_{i}\right)+\alpha_{0}\left(\Delta_{i+1}^{2}-\Delta_{i}^{2}\right)+\alpha_{3}\left(\Delta_{i+1-1}^{3}-\Delta_{i}^{3}\right),
$$

nelle quali

$$
\delta_{i+1, i}=T_{i+1}-1
$$

\section{Ponendo}

$$
\Delta_{i+1}-\Delta_{i}=a_{i}{ }_{i} ; \Delta_{i-i-i}^{2}-\Delta_{i}{ }^{2}=b_{i+1}, \Delta^{3}{ }_{i+1}-\Delta_{i}{ }^{3},-\delta_{i+1}, i=l_{i+1},
$$

si ottiene il seguente sistema di equazioni di condizione

$$
a_{i+1} \alpha_{1}+b_{i+1} \alpha_{2}+c_{i+1} \alpha_{3}+l_{i+1}=v_{i+1}
$$

nelle quali le $v_{\mathrm{i}+\mathrm{t}}$ rappresentano i residui dovuti agli errori di osservazione. Il metodo dei minimi quadrati consente una prima determinazione dei tre coefficienti $\alpha_{1}, \alpha_{2}, \alpha_{3}$, che, sostituiti nella [2] danno luogo al sistema di $n$ equazioni nella sola incognita $\alpha_{0}$ :

$$
\alpha_{\mathrm{o}}+g\left(\Delta_{\mathrm{i}}\right)-t_{\mathrm{i}}=v_{\mathrm{i}}^{\prime}
$$

essendo

$$
t_{1}=T_{i}-t_{0}, g\left(\Delta_{i}\right)=\alpha_{1} \Delta_{i}+\alpha_{2} \Delta_{i}{ }^{2}+\alpha_{3} \Delta_{i}{ }^{3} .
$$

Una ulteriore determinazione più approssimata dei coefficienti $\alpha_{1}, \alpha_{2}, \alpha_{3}$, si ottiene sostituendo nelle [2] il valore più probabile di $\alpha_{0}$ dedotto risolvendo il sistema [5]; si è cosi condotti al sistema

$$
\alpha_{1} \Delta_{i}+\alpha_{2} \Delta_{i}^{2}+\alpha_{3} \Delta_{i}^{3}-\left(T_{i}-t_{0}+\alpha_{0}\right)=v_{i}^{\prime \prime}
$$

clse, ponendo

$$
\lrcorner_{\mathrm{i}}=a_{\mathrm{i}}{ }^{\prime},\right\lrcorner_{\mathrm{i}}{ }^{2}=b_{\mathrm{i}}{ }^{\prime},\right\lrcorner_{\mathrm{i}}{ }^{3}=c_{\mathrm{i}}{ }^{\prime},-\left(t_{\mathrm{i}}+\alpha_{\mathrm{o}}\right)=l_{\mathrm{i}}^{\prime}
$$

può essere scritto nella forma usuale

$$
a_{i}^{\prime} \alpha_{1}+b_{i}^{\prime} \alpha_{2}+r_{i}^{\prime} \alpha_{3}+l_{i}^{\prime}=v_{i}^{\prime \prime} .
$$


Il sistema dei valori $\alpha_{1}, \alpha_{2}, \alpha_{3}$, che rendono minima la somma dei quadrati dei residui è, com'è noto, deducibile dal sistema normale

$$
\begin{aligned}
& {\left[a^{\prime} a^{\prime}\right] \alpha_{1}+\left[a^{\prime} b^{\prime}\right] \alpha_{2}+\left[a^{\prime} c^{\prime}\right] \alpha_{3}+\left[a^{\prime} l^{\prime}\right]=0} \\
& {\left[a^{\prime} b^{\prime}\right] \alpha_{1}+\left[b^{\prime} b^{\prime}\right] \alpha_{2}+\left[b^{\prime} c^{\prime}\right] \alpha_{3}+\left[b^{\prime} l^{\prime}\right]=0} \\
& {\left[a^{\prime} c^{\prime}\right] \alpha_{1}+\left[b^{\prime} c^{\prime}\right] \alpha_{2}+\left[c^{\prime} c^{\prime}\right] \alpha_{3}+\left[c^{\prime} l^{\prime}\right]=0}
\end{aligned}
$$

dove, per il noto significato dei simboli,

$$
\begin{aligned}
& {\left[a^{\prime} a^{\prime}\right]=\Sigma_{\mathrm{i}} \Delta_{\mathrm{i}}^{2},\left[a^{\prime} b^{\prime}\right]=\Sigma_{\mathrm{i}} \Delta_{\mathrm{i}}^{3},\left[a^{\prime} c^{\prime}\right]=\Sigma_{\mathrm{i}} \Delta_{\mathrm{i}}^{4},\left[a^{\prime} l^{\prime}\right]=\Sigma_{\mathrm{i}} \Delta_{\mathrm{i}}\left(a_{\mathrm{o}}-t_{\mathrm{i}}\right),} \\
& {\left[b^{\prime} b^{\prime}\right]=\left[a^{\prime} c^{\prime}\right]=\Sigma_{\mathrm{i}} \Delta_{\mathrm{i}}^{4},\left[b^{\prime} c^{\prime}\right]=\Sigma_{\mathrm{i}} \Delta_{\mathrm{i}}^{5},\left[b^{\prime} l^{\prime}\right]=\Sigma_{\mathrm{i}} \Delta_{\mathrm{i}}^{2}\left(a_{\mathrm{o}}-t_{\mathrm{i}}\right),} \\
& {\left[c^{\prime} c^{\prime}\right]=\Sigma_{\mathrm{i}} \Delta_{\mathrm{i}}{ }^{\circ},\left[c^{\prime} l^{\prime}\right]=\Sigma_{\mathrm{i}} \Delta_{\mathrm{i}}^{3}\left(a_{\mathrm{o}}-t_{\mathrm{i}}\right) .}
\end{aligned}
$$

Evidentemente l'equazione della dromocrona così dedotta si potrà considerare sufficientemente valida per distanze comprese nell'intervallo relativamente al quale si dispone di un sufficiente numero di dati sperimentali.

Sono state esaminate le registrazioni originali o copie fotografiche delle 66 stazioni d'osservazione elencate nella Tabella $I$, nella quale sono riportati: a) nelle colonne (1) e (2) le distanze epicentrali e gli azimut delle singole stazioni dall'epicentro, calcolati servendosi delle note formule ed adoperando latitudini geocentriche; $b$ ) nella colonna (3) i tempi d'inizio delle registrazioni; c) nella colonna (4) i corrispondenti tempi di propagazione riferiti all'ora epicentrale.

Le stazioni, i cui dati, particolarmente sicuri, sono stati utilizzati in precedenza per la determinazione dell'epicentro e dell'ora origine, sono contraddistinte col segno ( $)$. La fig. 1 riproduce un planisfero (in proiezione di Mercatore) sul quale sono state tracciate le linee isodistanti, intervallate di $10^{\circ}$, e le linee isoazimutali, intervallate di $22^{\circ}, 5$ allo scopo di caratterizzare la distribuzione delle varie stazioni utilizzate rispetto all'epicentro.

Per una prima determinazione approssimata dei coefficienti $a_{0}$, $a_{1}, a_{0}$ sono stati utilizzati i tempi di registrazione delle 40 stazioni che, nella citata tabella, sono contradistinte col segno ("). Per evitare risultati non sufficientemente attendibili, sono stati esclusi dal calcolo, oltre che i dati relativi a quelle stazioni le cui distanze superano il limite di percettibilità delle $P$, anche i dati che, riportati in un primo grafico tracciato a scopo orientativo, si sono manifestati netlamente 
Tabella I

\begin{tabular}{|c|c|c|c|c|c|c|}
\hline \multirow[t]{2}{*}{ S T A Z I O N I } & \multirow[t]{2}{*}{$\begin{array}{l}1 \\
1\end{array}$} & \multirow[t]{2}{*}{$\begin{array}{c}2 \\
\text { Aximut }\end{array}$} & \multirow{2}{*}{$\begin{array}{c}3 \\
\text { Tempi di } \\
\text { registraz. } \\
(01 \text { h. }+) \\
\end{array}$} & \multicolumn{2}{|c|}{$\begin{array}{cc}4 & 5 \\
\text { Tempi di } & \text { tragitto }\end{array}$} & \multirow[t]{2}{*}{$\begin{array}{c}6 \\
O-C\end{array}$} \\
\hline & & & & osservati & calcolati & \\
\hline & $\circ$ & o & m. s. & m. s. & m. s. & $\mathbf{s}$ \\
\hline 1. Ambulong & 34.265 & 22111.4 & $29+4.0$ & $702.5 ?$ & & \\
\hline 2. College (') (") & 43.712 & 3509.9 & 3050.5 & 809.0 & 808.4 & +0.6 \\
\hline 3. Shillong (') (") & 45.273 & 26512.5 & 3102.1 & 820.6 & 821.0 & -0.4 \\
\hline 4. Alipore & 49.679 & $26+30.5$ & 3212.2 & $930.7 ?$ & 835.5 & \\
\hline $\begin{array}{l}\text { 5. Sitka } \\
\text { 6. New Delhi (') (") }\end{array}$ & $\begin{array}{l}51.309 \\
5+434\end{array}$ & $\begin{array}{r}4346.4 \\
27806.2\end{array}$ & $\begin{array}{ll}32 & 03.5 \\
32 & 12.1\end{array}$ & $\begin{array}{l}922.0 ? \\
930.6\end{array}$ & $\begin{array}{l}907.7 \\
930.8\end{array}$ & -0.2 \\
\hline 7. Resolute Bay (") (") & 56.973 & 1540.0 & 3229.2 & 947.7 & 949.0 & -1.3 \\
\hline 8. Victoria & 61.597 & 49.00 .6 & 3329.7 & $1048.2 ?$ & 1020.9 & \\
\hline 9. Kiruna (") (") & 62.048 & $33853 . t$ & 3304.8 & 1023.3 & 1023.9 & -0.6 \\
\hline 10. Seattle & 62.691 & +921.5 & $3321 . t$ & 1039.9 ? & 1028.3 & \\
\hline 11. Poona (") & 62.828 & $270+6.6$ & בـ 33 & 1028.0 & 1029.1 & -1.1 \\
\hline 12. Bombay (') & 63.368 & 27147.0 & 3314.5 & 1033.0 & 1032.7 & +0.3 \\
\hline 13. Kodaikanal & 65.506 & 26116.2 & $33+0.0$ & $1058.5 ?$ & 1046.6 & \\
\hline 14. Upsala (') (") & 68.812 & 33359.0 & 3348.6 & 1107.1 & 1107.5 & -0.4 \\
\hline 15. Mt. Hamilton (') (") & 69.139 & 5811.9 & 3352.1 & 1110.6 & 1109.5 & +1.1 \\
\hline 16. Fresno (') & 70.680 & 5742.2 & 3400.4 & 1118.9 & 1119.0 & -0.1 \\
\hline 17. Reykiavik (") (") & 73.142 & 35322.3 & $3+15.0$ & 1133.5 & 1133.7 & -0.2 \\
\hline 18. Boulder City (") (") & 74.237 & 55385 & $3+22.4$ & $11+0.9$ & 1140.1 & +0.8 \\
\hline 19. Praha (") (") & 77.677 & 329115 & 3442.1 & 1200.6 & 1159.7 & +0.9 \\
\hline 20. Budapest (') & 77.869 & 32506.1 & $3+43.9$ & 1202.4 & 1200.8 & +1.6 \\
\hline 21. Jena (') (") & 78.017 & 33113.6 & $3+43.1$ & 1201.6 & 1201.6 & 0.0 \\
\hline 22. Kecskemet & $78.0+2$ & $33+23.5$ & 3506.0 & $1221.5 ?$ & 1201.7 & \\
\hline 23. Gottingen (') (") & 78.068 & 33226.7 & $34+4.2$ & 1202.7 & 1201.9 & +0.8 \\
\hline 24. Szeged & 78.373 & $323+2.9$ & 3459.3 & $1217.8 ?$ & 1203.6 & \\
\hline 25. Vienna (') (") & 78.385 & 32702.5 & $34+6.5$ & 1205.0 & 1203.7 & +1.3 \\
\hline 26. Kalocsa & 78.548 & 32432.5 & 3459.0 & $1217.5 ?$ & 1203.9 & \\
\hline 27. De Bilt & 79.157 & 33518.7 & 3500.0 & 1218.5 ? & 1208.1 & \\
\hline 28. Beograd (') (") & 79.339 & 32237.7 & $3+50.4$ & 1208.9 & 1208.9 & 0.0 \\
\hline 29. Stuttgart (') (") & 80.674 & 331.19 .9 & 3458.5 & 1217.0 & 1216.2 & +0.8 \\
\hline 30. Rathfarnham (') & 81.156 & $3+212.2$ & 3502.5 & 1221.0 & 1213.8 & +2.2 \\
\hline 31. Kew (') (") & 81.327 & 33804.1 & 3502.0 & 1220.5 & 1219.7 & +0.8 \\
\hline 32. Strasbourg (') (") & 81.335 & 33202.3 & 3502.0 & 1220.5 & 1219.7 & +0.8 \\
\hline 33. Basel (') (") & 82.289 & 33135.9 & 3507.2 & 1225.7 & 1224.8 & +0.9 \\
\hline 34. Neuchatel ( $\left.{ }^{\prime}\right)\left({ }^{\prime \prime}\right)$ & 82.978 & $331+1.3$ & 3510.4 & 1228.9 & 1228.1 & +0.5 \\
\hline 35. Padova (') (") & 83.241 & 32723.6 & 3510.7 & 1229.2 & 1229.8 & -0.6 \\
\hline 36. Bologna & 83.462 & 32743.7 & 3515.2 & 1233.7 & 1231.1 & +2.6 \\
\hline 37. Pavia & 83.717 & 32924.5 & 3522.7 & $12+1.2 ?$ & 1233.3 & \\
\hline 38. Auckland & 83.934 & 15513.7 & 3536.5 & $1255.0 ?$ & 1232.3 & \\
\hline 39. Chicago U.S.C.G.S. (') (") & 84.334 & $35+7.5$ & 3515.0 & 1233.5 & 1235.5 & -2.0 \\
\hline 40. Helwan (') (") & 84.807 & 30607.6 & 3520.0 & 1238.5 & 1237.9 & +0.6 \\
\hline 41. Roma (') (") & 85.124 & 32533.8 & 3521.0 & 1239.5 & 1239.6 & -0.1 \\
\hline 42. Clermont (') (") & 85.368 & 33324.3 & 3522.2 & 1240.7 & 124.0 .8 & -0.1 \\
\hline 43. Shawinigan Falls & 85.721 & 2409.8 & 3536.0 & $1254.5 ?$ & $12+2.5$ & \\
\hline 44. Seven Falls & 85.779 & $22+3.1$ & 3537.7 & $1256.2 \%$ & 1242.6 & \\
\hline 45. Ottawa (") (") & 85.819 & 2631.9 & 3523.4 & $12: 1.9$ & $12+3.1$ & -1.2 \\
\hline 46. Messina (') (") & 86.828 & 32130.9 & 3529.0 & $12+7.5$ & 1248.2 & $-0 . \bar{\imath}$ \\
\hline 47. Cleveland (') (") & 87.098 & 3209.5 & 3530.5 & $12+9.0$ & $12+9.5$ & -0.5 \\
\hline 48. Cincinnati (") (") & 87.856 & 3519.8 & 3534.8 & 1253.3 & 1253.3 & 0.0 \\
\hline 49. Wellington & 87.996 & 15657.3 & $35+2.0$ & $1300.5 ?$ & 1253.9 & \\
\hline 50. State College (') (") & 89.095 & 3007.3 & $35+0.5$ & 1259.0 & 1259.3 & -0.3 \\
\hline 51. Christchurch (') (") & 89.484 & 15916.4 & $35+1.3$ & 1259.8 & 1301.2 & -1.4 \\
\hline 52. Harvard (") (") & 89.742 & 2508.6 & 3543.8 & 1302.3 & 1302.5 & -0.2 \\
\hline 53. Halifax & 89.988 & 1900.6 & 3603.8 & $1322.3 ?$ & 1303.7 & \\
\hline 54. Palisades (') (") & $90.32+$ & 2722.3 & 3545.3 & 1303.8 & 1305.3 & -1.5 \\
\hline 55. Tortosa & 90.628 & 33249.5 & 3556.0 & $1314.5 ?$ & 1306.6 & \\
\hline 56. Washington D.C. (') (") & 91.043 & 3029.0 & $35+9.2$ & 1307.7 & 1308.7 & -1.0 \\
\hline 57. Algeri Univ. (") (") & 93.305 & 32911.4 & 3558.6 & 1317.1 & 1319.3 & -2.2 \\
\hline 58. Coimbra & 93.901 & $338+9.5$ & 3623.8 & $13+2.3 ?$ & 1322.0 & \\
\hline 59. Cartuja & 95.261 & 33410.3 & 3626.0 & $1344.5 ?$ & I 328.3 & \\
\hline 60. Lisbona & $95 .+25$ & $338+9.8$ & 3621.5 & $1340.0 ?$ & 1329.2 & \\
\hline 61. Malaga & 95.946 & 33435.3 & 3623.2 & $1341.7 ?$ & 1331.1 & \\
\hline 62. Bermuda & 101.174 & 2402.1 & 3644.9 & $1403.4 ?$ & 1353.7 & \\
\hline 63. Tamanrasset (') & 104.396 & 32022.3 & 3650.6 & 1409.1 & 1406.8 & +2.3 \\
\hline 64. San Juan & 113.595 & 3048.7 & 3801.5 & 1520.0 & & \\
\hline 65. Johannesburg & 125.498 & 27436.5 & $\$ 354.1$ & 2112.6 & & \\
\hline 66. La Plata & $161.68+$ & 7250.2 & 4300.0 & 2018.5 & & \\
\hline
\end{tabular}




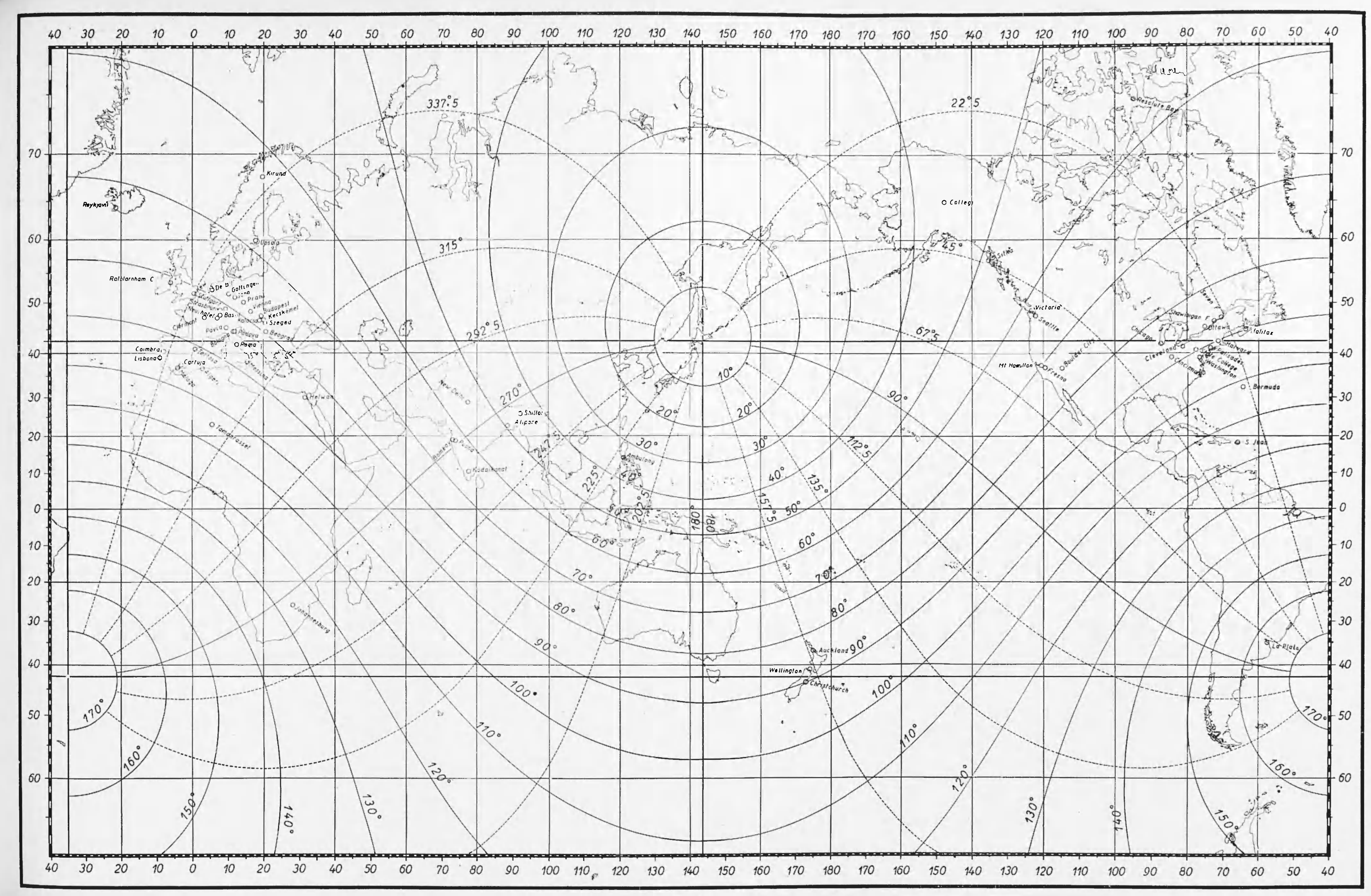




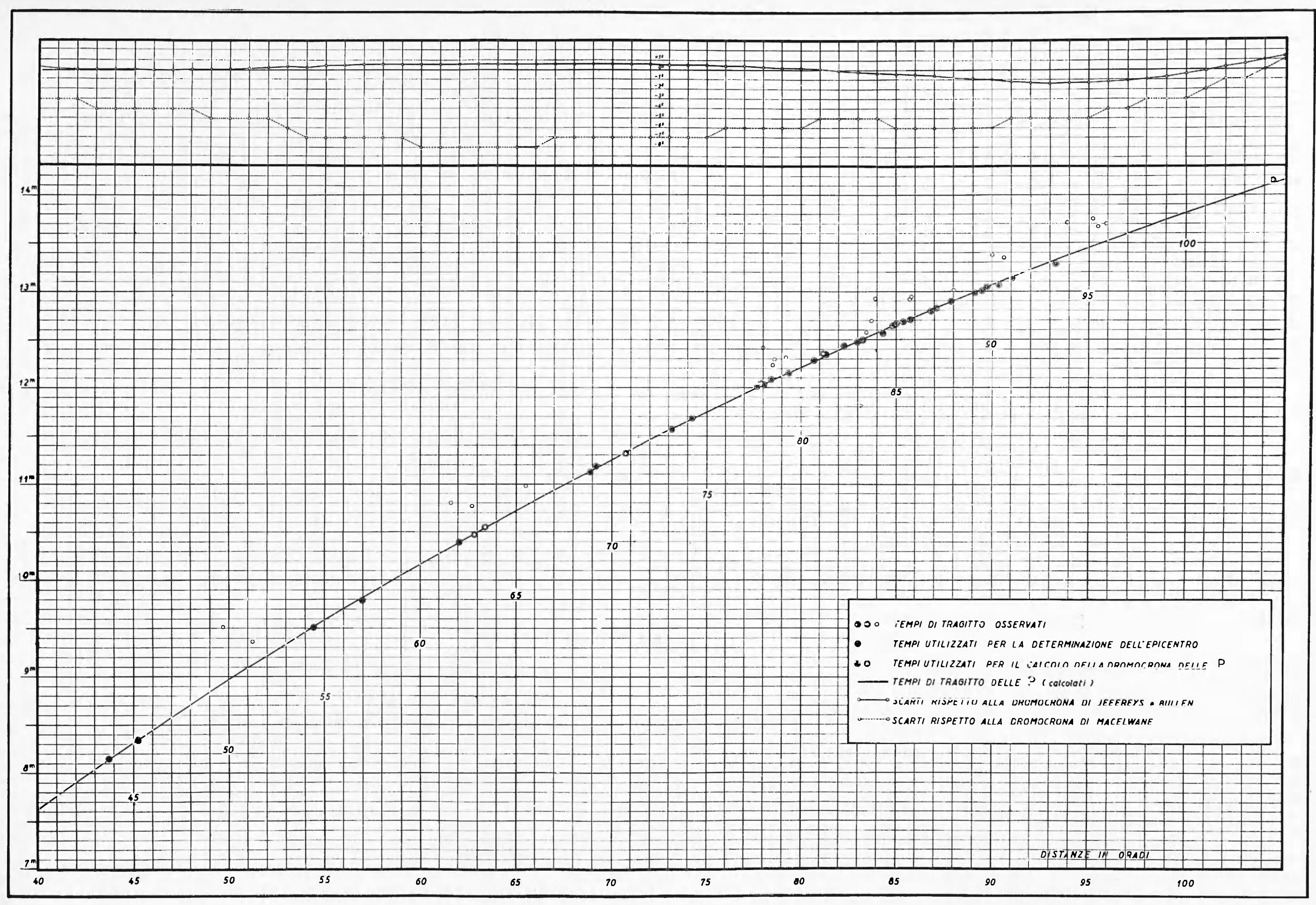


discordanti dall'andamento, sia pure grossolanamente regolare, dei rimanenti dati sperimentali.

La risoluzione del sistema delle corrispondenti 39 equazioni di condizione del tipo [4], hat dato i seguenti risultati:

$$
\begin{aligned}
& u_{1}=12,2525625, \\
& u_{1}=-5,5531466 \cdot 10^{-2}, \\
& \varkappa_{3}=0,1144524 \cdot 10^{-3},
\end{aligned}
$$

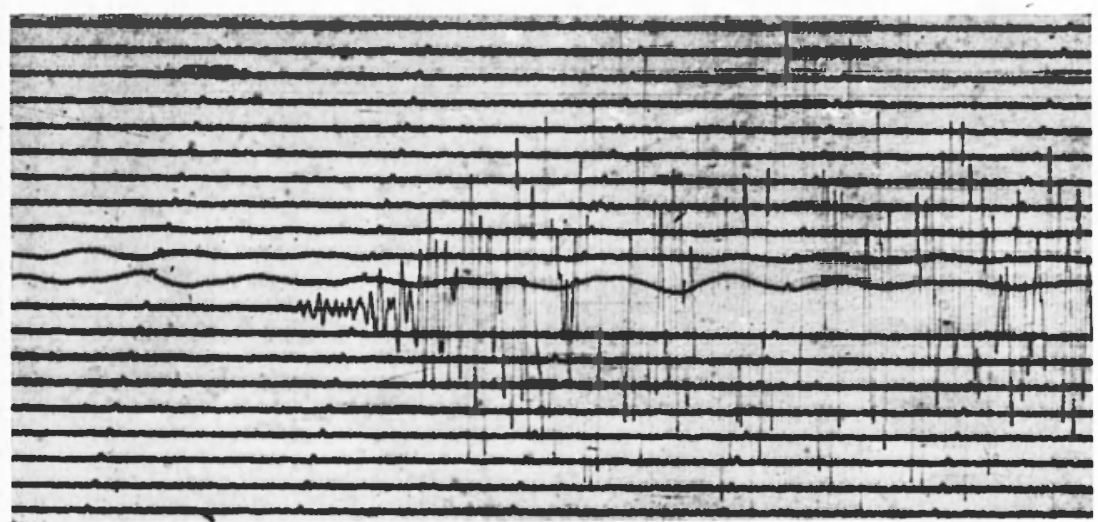

Fig. 3 - Boulder City $\left(1=7 t^{\prime \prime} .24 ; \alpha 5.5^{\prime \prime} 38^{\prime} .5\right)$

con l'errore medio

$$
\varepsilon= \pm 1.106898 \text {. }
$$

La somma dei quadrati dei residui è risultata

$$
[v v]=44,1080
$$

sensibilmente coincidente con

$$
[l l \cdot 3]=44,1088 .
$$

In hase a tali risultali si sono calcolati, per ormma delle 40 stazioni utilizzate, $\mathrm{i}$ valori del trinomio

$$
g\left(\Delta_{i}\right)=\alpha_{1} \Delta_{i}+\alpha_{2} \Delta_{i}^{2}+\alpha_{3} \Delta_{i}^{3}
$$

riportati, unitamente alle differenze $g\left(J_{1}\right)-t_{1}$, nella Tabella II, che hamno consentito, conformemente alle [5], la determinazione del ralore piì probalbile del coefficiente $\alpha_{0}$. Si te ottenuto

$$
\alpha_{0}=48,719 \pm 0,272 \text {. }
$$


Tabella II

1. College

2. Shillong

3. New Delhi

4. Resolute Bay

5. Kiruna

6. Poona

7. Bombay

8. Upsala

9. Mt. Hamilton

10. Fresno

11. Reykjavik

12. Boulder City

13. Praha

14. Budapest

15. Jena

16. Gottingen

17. Vienna

18. Beograd

19. Stuttgart

20. Rathfarnliam

21. Kew

22. Strasbourg

23. Basel

24. Neuchatel

25. Padova

26. Chicago

27. Helwan

28. Roma

29. Clermont

30. Ottawa

31. Messina

32. Cleveland

33. Cincinnati

34. State College

35. Christchurch

36. Harvard

37. Palisades

38. W/ashington D.C.

39. Algeri Univ.

40. Tamanrsset
439.03731

451.50106

520.87322

538.98023

573.79417

578.98633

582.55668

617.46911

619.50485

629.00724

643.88180

650.37834

670.32306

671.41587

672.25679

672.54628

674.34229

679.71269

687.14157

689.79930

690.73909

690.78302

695.99613

699.73023

701.14881

707.00444

709.51876

711.19721

712.48594

714.85862

720.12950

721.53103

725.44578

731.78206

733.75557

735.06033

737.99156

741.58987

752.74781

804.12738
$-49.96269$

$-49.09894$

$-49.72678$

$-48.71977$

$-49.50583$

$-49.01367$

$-50.44332$

$-49.63089$

$-51.09515$

$-49.89276$

$-49.61820$

$-50.52166$

$-50.27694$

$-50.98413$

$-49.34321$

$-50.15372$

$-50.65771$

$-49.18731$

$-49.85843$

$-51.20070$

$-49.76091$

$-49.71700$

$-49.70387$

$-49.16977$

$-48.05119$

$-46.49556$

$-48.98124$

$-48.30279$

$-48.21406$

$-47.04138$

$-47.37050$

$-47.46897$

$-47.85422$

$-47.21794$

$-46.04443$

$-47.23967$

$-45.80844$

$-46.11013$

$-4.35219$

$-4.97262$ 
Passando successivamente alla risoluzione del sistema $\left[6^{\prime}\right]$, ottenuto dal sistema [2] sostituendo il valore di $\alpha_{0}$ cosi determinato, si sono ottenuti i seguenti valori più approssimati dei coeflicienti $a_{1}$, $\alpha_{n}, \alpha_{\infty}$, ed i corrispondenti errori medi :

$$
\begin{aligned}
& \alpha_{1}=12,1460454+0,063488, \\
& \alpha_{2}=-5,1065838 \cdot 10^{2} \div 0,163986 \cdot 10^{2}, \\
& \alpha_{:}=0,0761058 \cdot 10^{3}+0,01056 \cdot 10^{3} .
\end{aligned}
$$

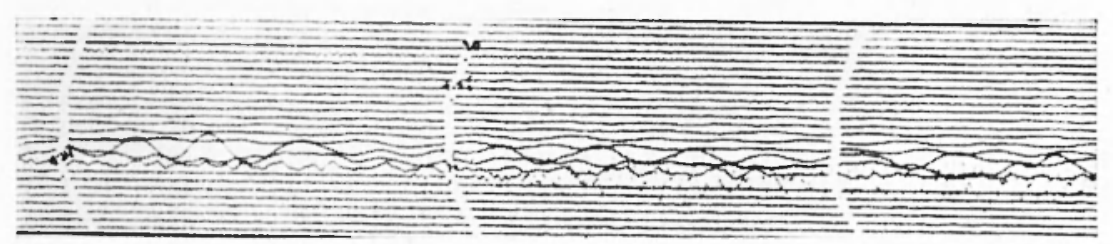

Fig. 1. Stuttgart $\left(\Delta=80 " .67 ;\right.$ a $\left.331^{\circ} 19^{\prime} .9\right)$

Per il consueto controllo si è ottenuto:

$$
\begin{aligned}
& {\left[v^{\prime \prime} v^{\prime \prime}\right]=41,62312} \\
& {\left[l l^{\prime} \cdot 3\right]=41,62313}
\end{aligned}
$$

In definitiva si può ritenere che l'equazione della dromocrona più probahile delle $P$, valevole per il terremoto oggetto di questo stu. dio e per distanze comprese tra $40^{\circ}$ e $105^{\circ}$, è la seguente:

$$
\begin{aligned}
& I=\left(0,0761 \cdot 10^{-3}+0,0106 \cdot 10^{-3}\right) \Delta^{3}-\left(5,106584 \cdot 10^{-2}+0,163986 \cdot 10^{2}\right) \\
& +(12,146045 \div 0,63488, د+48,719 .
\end{aligned}
$$

Nella Tabella III, sono riportati i tempi di tragitto calcolati di decimo in decimo di grado servendosi della [7]. Da un confronto con le tabelle di Jeffreys e Bullen ( $\left.{ }^{3}\right)$, di Gutenberg e Richter ( $\left.{ }^{4}\right)$ e di Macelwane (5), si nota che (Tabella IV) le deviazioni rispetto ai tempi di tragitto tahulati dai primi due autori superano di poco il secondo solo per distanze comprese tra $90^{\circ}$ e $105^{\circ}$; gli scarti rispetto ai tempi di Gutenberg e Richter si distribuiscono in un intervallo che va da $-3^{\mathrm{s}}$ a $+1^{\mathrm{s}}$, mentre gli scarti rispetto ai tempi di Macelwane (Tabella IVc) si distribuiscono nell'intervallo $-8^{s}$, $+1^{\text {s, }}$, analogamente a quanto già osservato da Gutenberg e Richter ( $\left.{ }^{4}\right)$. 


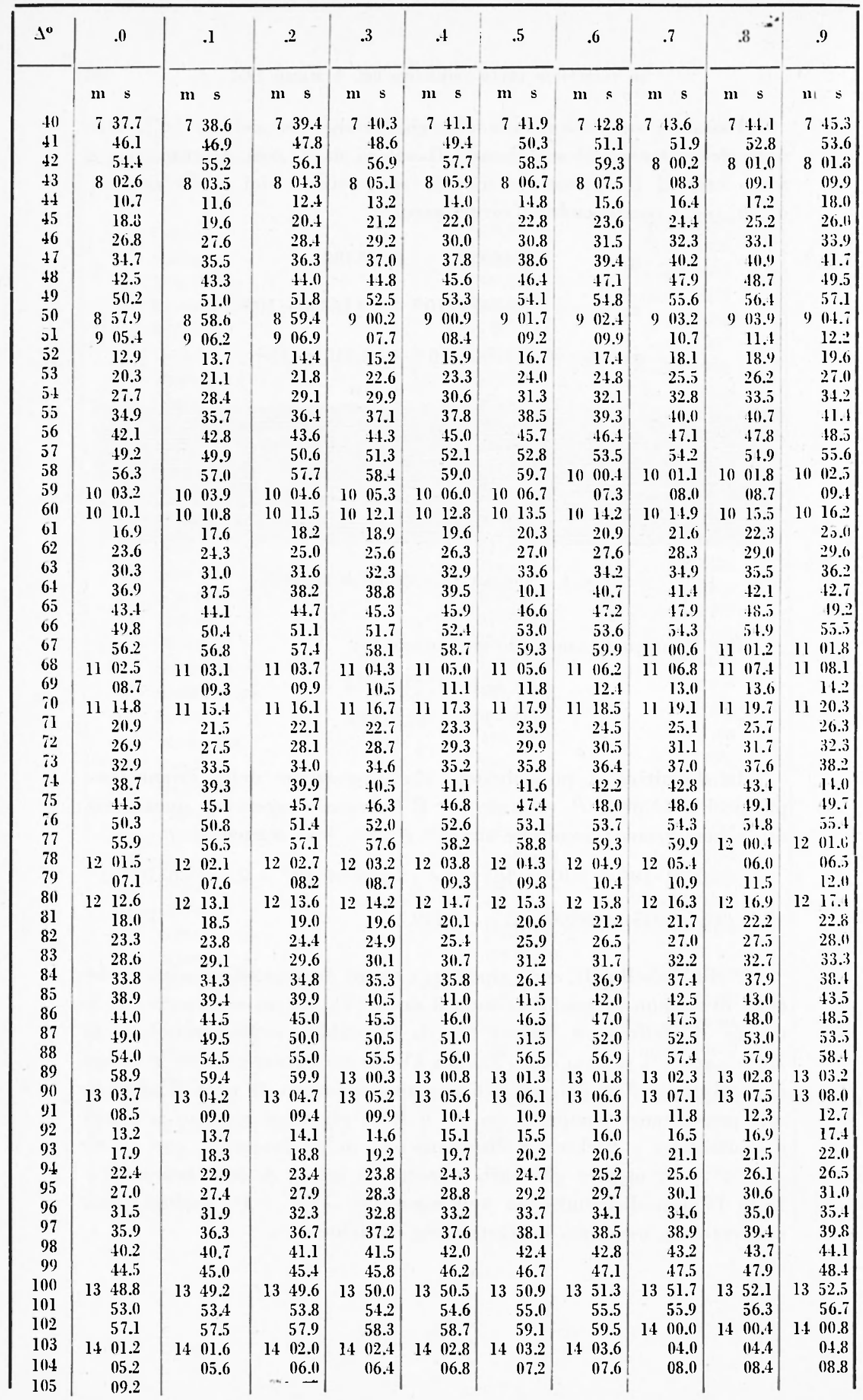


Nella Talella I sono anche riportati, nella colonna (5) i tempi di tragilto, relativi alle singole stazioni di osservazione, calcolati mediante la (7) e nella colonna (6) le differenze tra i tempi osservati e quelli calcolati. Si nota chiaramente che, mentre per il gruppo delle 40 stazioni, i cui dati sono stati utilizzati nei calcoli precedenti, tali diffe-

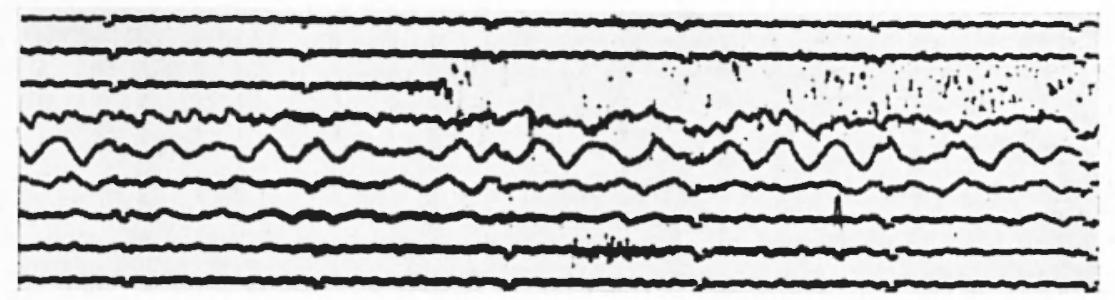

Fig. 5 - Cleveland $\left(\Delta=87^{\circ} .10 ; \alpha=32^{\circ} 09^{\circ} .5\right)$

renze sono dell'ordine prevedibile dalla teoria degli errori d'osservazione, per il rimanente gruppo delle 19 stazioni si hanno differenze sistematicamente positive (fig. 2 ), il che prova che $\mathbf{i}$ tempi rilevati in queste ultime appartengono ad impulsi successivi a quelli dovuti al vero inizio delle $P$. A tale proposito è da notare che, nei sismogrammi esaminati indipendentemente dalla distanza e dall'azimut, le registrazioni nonostante la eccezionale intensità dello scuotimento sismico, iniziano con una lieve emersio seguita, ad una decina di secondi, da bruschi ed ampi impulsi che danno alla parte iniziale delle registrazioni un aspetto particolare. Tale aspetto in alcuni sismogrammi è talmente marcato che può fare pensare alla registrazione successiva di scosse distinte. Presumililmente queste caratteristiche sono da attri-

TABELla IV-a

Scarti rispetto ai tempi tragitto calcolati da Jeffreys e Bullen.

\begin{tabular}{|c|c|c|c|c|c|c|c|c|c|c|}
\hline & ) & 1 & 2 & 3 & 4 & 5 & 6 & 7 & 8 & 9 \\
\hline $40 "$ & 0.4 & 0.2 & 0.1 & 0.1 & 0.1 & 0.1 & 0.0 & 0.0 & 0.1 & 0.1 \\
\hline $50 "$ & 0.1 & 0.2 & 0.3 & 0.4 & 0.3 & 0.5 & 0.5 & 0.6 & 0.6 & 0.6 \\
\hline$\delta\left(k^{\prime}\right)$ & 0.6 & 0.6 & 0.7 & 0.6 & 0.6 & 0.6 & 0.6 & 0.6 & 0.6 & 0.6 \\
\hline $70^{\circ}$ & 0.6 & 0.6 & 0.6 & 0.6 & 0.5 & 0.5 & 0.1 & 0.4 & 0.3 & 0.2 \\
\hline $80^{\circ}$ & 0.2 & 0.1 & -0.1 & -0.2 & -0.3 & -0.4 & -0.5 & -0.7 & -0.8 & -0.9 \\
\hline 90. & -1.0 & -1.2 & -1.3 & -1.4 & -1.3 & -1.3 & -1.2 & -1.1 & -0.9 & -0.7 \\
\hline $100^{\circ}$ & -0.4 & -0.1 & -0.3 & 0.6 & 1.0 & 1.1 & & & & \\
\hline
\end{tabular}


TaBella IV-b

Scarti rispetto ai tempi di tragitto calcolati da Gutenberg e Richter.

\begin{tabular}{|r|r|r|r|r|r|r|r|r|r|r|}
\hline & 0 & 1 & 2 & 3 & 4 & 5 & 6 & 7 & 8 & 9 \\
\hline $40^{\circ}$ & 0 & 1 & 1 & 1 & 1 & 1 & 0 & -1 & 0 & 0 \\
$50^{\circ}$ & -1 & -1 & -2 & -1 & -2 & -2 & -2 & -1 & 0 & 0 \\
$60^{\circ}$ & 0 & 0 & 0 & 0 & 0 & 1 & 0 & 1 & 1 & 0 \\
$70^{\circ}$ & 0 & 0 & 0 & -1 & -1 & -2 & -1 & -1 & -1 & 0 \\
$80^{\circ}$ & -1 & -1 & -1 & -2 & -2 & -2 & -2 & -2 & -2 & -2 \\
$90^{\circ}$ & -2 & -1 & -2 & -3 & -2 & -3 & -2 & -3 & -3 & -3 \\
$100^{\circ}$ & -2 & -2 & -2 & -1 & 0 & 0 & & & &
\end{tabular}

\section{TABella IV-c}

Scarti rispetto ai tempi di tragitto calcolati da Maceluane.

\begin{tabular}{|r|r|r|r|r|r|r|r|r|r|r|}
\hline & 0 & 1 & 2 & 3 & 4 & 5 & 6 & 7 & 8 & 9 \\
\cline { 1 - 3 } & & & & & & & & & \\
\hline $40^{\circ}$ & -3 & -3 & -3 & -4 & -4 & -4 & -4 & -4 & -4 & -5 \\
$50^{\circ}$ & -5 & -5 & -5 & -6 & -7 & -7 & -7 & -7 & -7 & -7 \\
$60^{\circ}$ & -6 & -8 & -8 & -8 & -8 & -8 & -8 & -7 & -7 & -7 \\
$70^{\circ}$ & -7 & -7 & -7 & -7 & -7 & -7 & -6 & -6 & -6 & -6 \\
$80^{\circ}$ & -6 & -5 & -5 & -5 & -5 & -6 & -6 & -6 & -6 & -6 \\
900 & -6 & -5 & -5 & -5 & -5 & -5 & -4 & -4 & -3 & -3 \\
100 & -3 & -2 & -1 & -1 & 0 & 1 & & & &
\end{tabular}

buirsi ad un particolare meccanismo di produzione all'ipocentro che ha dato luogo ad un processo, di una certa durata, nel corso del quale le vibrazioni a breve periodo possono essere state modificate per un fenomeno d'interferenza.

Istituto Nazionale di Geofisica - Osserv. di Messina - Ottobre 1953.

\section{RIASSUNTO}

A proseguimento dello studio dedicato al terremoto dello Hokkaido del 4 marzo 1952, viene dedotta, mediante un metodo di approssimazioni successive l'equazione della dromocrona più probabile delle onde $\mathrm{P}$, valevole per distanze comprese tra $40^{\circ}$ e $105^{\circ}$. In base a tale 
equazions vengono calcolati i tempi di tragitto e viene istituito un confronto con gli analoghi tempi calcolati da Jeffreyes e Bullen, da Gutenberge Richter "da Maceluane. Viene notata la coincidenza, nei limiti degli errori sperimentali, con i tempi di Jeffreys e Bullen.

Si giustifica il notevole ritardo riscontrato nellinizio delle registrazioni in alcune stazioni.

\section{SUMMARY}

For the advancement of the study of the earthquate of Hohkaido of March 4, 1952, there has been deduced, by means of a method of successive approximations, the equation of the most probable dromochrone of the $\mathrm{P}$ wave, valid for distances included between $40^{\prime \prime}$ and 105". On the basis of such an equation, the passage times have been calcolated and a comparison has been made between the analogous times calcolated by Jeffreys and Bullen, by Gutenberg and Richter, and by Macelwane. A coincidenes is pointed out, within the limits of the experimental errors, with the times of Jeffreys and Bullen.

The considerable retard noted in the begins of the registrations in various stations has been justified.

\section{BIBLIOGRAFIA}

(1) Gimaxm A. Il terremoto dello Holkitrido del 4 marzo 1952. Annali di Geo. fisieri VI, 2. 1953.

1-1 Cinol P. - Peronict F., $1 /$ terremoto del Turkestan del 2 novembre 1946. Anmali di Geofisica I, 2, 1948.

(3) Jefrews H. Bullax K. E., Seismological Tables. British Association for the Advancement of Science: Gray Milne Trust. London 1910.

(t) Gotenbers: B. and Richten C. F., On seismic unes ifirst Paperi. Gerl. Beitr. \%. Geopli., Band 13, 1935.

(i) Macelwaxe J. B., A Preliminary Table o of observed travel times of earthquake uaves for distances between 10" and 180", applicable only to normal Earthquakes. Saint Louis Iniversity, 1933. 